\title{
SUMMABILITY $C$ OF SERIES OF SURFACE SPHERICAL HARMONICS $\left({ }^{1}\right)$
}

\author{
BY \\ AARON SIEGEL
}

I. Introduction. 1.1. SURface SPHERICAL harmonics. Let $\Omega$ denote the surface of the unit sphere in Euclidean 3-space, whose center is the origin $O$ of a system of Cartesian coordinates $x, y, z$. Let $Q$ denote a point on $\Omega$. The function $Y_{n}(Q)$ is said to be a surface spherical harmonic of degree $n$ if $H_{n}(x, y, z)$ is a homogeneous harmonic polynomial of degree $n$ and $H_{n}(x, y, z)$ $=r^{n} Y_{n}(Q)$ where $(x, y, z)$ lies on the line through $O$ and $Q$ at a distance $r$ from $O$.

1.2. LAPLACE SERIES. If $f(Q)$ is a Lebesgue integrable function on $\Omega$, the Laplace series of $f(Q)$ is a series of surface spherical harmonics $\sum_{n=0}^{\infty} Y_{n}(Q)$ where $Y_{n}(Q)$ is defined by $Y_{n}(Q) \equiv[(2 n+1) / 4 \pi] \iint_{\Omega} f(M) P_{n}([M, Q]) d \Omega_{M}$. Here $[M, Q]$ denotes the inner product of the unit vectors $O M$ and $O Q$ and $P_{n}([M, Q])$ denotes the Legendre polynomial of order $n$.

In this paper necessary and sufficient conditions for the Cesaro summability of series of surface spherical harmonics are obtained. The analogous results for trigonometric series were given by Plessner [11, p. 256]. In the field of Laplace series sufficient conditions for Cesaro summability was obtained by Gronwall $[4$, p. 213] and by Fejer [3, p. 267] and a necessary and sufficient condition for the convergence of a particular class of Laplace series were obtained by V. L. Shapiro [9, p. 514]. The latter also obtained sufficient conditions for the Cesaro summability of series of surface spherical harmonics $[8$, p. 212].

II. Generalized Laplacians. 2.1. Definition. For a point $P$ on $\Omega$ let $C\left(P, h^{\prime}\right)$ denote the circle of intersection of $\Omega$ and the sphere of radius $2 \sin \left(h^{\prime} / 2\right), 0<h^{\prime}<\pi$, whose center is at $P$. Let $f(Q)$ be a function defined in the neighborhood $D\left(P, h^{\prime}\right) \equiv\left\{Q \in \Omega \mid[Q, P] \geqq \cos h^{\prime}\right\}$ of $P$, and integrable on the circumference of every circle $C(P, h)$ contained in this neighborhood. If $[1 / 2 \pi \sin h] \int_{C_{(P, h)}} F(Q) d s_{Q}$ has an expansion of the form:

$$
\begin{array}{r}
\frac{1}{2 \pi \sin h} \int_{C(P, h)} f(Q) d s_{Q}=\alpha_{0}+\alpha_{1} \frac{(1-\cos h)}{2}+\frac{\alpha_{2}}{(2 !)^{2}} \frac{(1-\cos h)^{2}}{2^{2}}+ \\
\cdots+\frac{\alpha_{r}}{(r !)^{2}} \frac{(1-\cos h)^{r}}{2^{r}}+o(1-\cos h)^{r}, \quad r=0,1, \cdots,
\end{array}
$$

Presented to the Society, April 23, 1960; received by the editors July 11, 1961.

(1) Submitted in partial fulfillment of the requirements for the degree of Doctor of Philosophy in the Graduate School of Arts and Sciences of Rutgers University. The author wishes to acknowledge his indebtedness to Professor V. L. Shapiro, under whose direction this thesis was written. 
we say that $f(Q)$ has an $r$ th generalized Laplacian at the point $P$ and denote it by $\triangle_{r} f(P)$. This generalized Laplacian is defined from the expansion (2.1.1) by setting $\Delta_{0} f(P) \equiv \alpha_{0}, \Delta(\Delta+1 \cdot 2) \cdots[\Delta+(k-1) k] f(P) \equiv \alpha_{k}, k=1,2, \cdots, r$, where $\Delta \cdot \Delta \cdot \cdots \cdot \Delta(k$ times $) \equiv \Delta_{k}$ and $\Delta_{k} \cdot \Delta_{j} \equiv \Delta_{k+j}$ for $j, k \geqq 0$ and $j+k \leqq r$. Thus if $[1 / 2 \pi \sin h] \int_{C(P, h)} f(Q) d s_{Q}$ has the expansion (2.1.1) then:

$$
\begin{aligned}
& \frac{1}{2 \pi \sin h} \int_{C(P, h)} f(Q) d s_{Q} \\
& =\Delta_{0} f(P)+\Delta_{1} f(P) \frac{(1-\cos h)}{2}+\frac{\Delta(\Delta+1 \cdot 2) f(P)}{(2 !)^{2}} \frac{(1-\cos h)^{2}}{2^{2}}+\cdots \\
& \quad+\frac{\Delta(\Delta+1 \cdot 2) \cdots[\Delta+(r-1) r] f(P)}{(r !)^{2}} \frac{(1-\cos h)^{r}}{2^{r}} \quad r=0,1, \cdots . \\
& \left.\quad+o(1-\cos h)^{r}, \quad r=0.2\right)
\end{aligned}
$$

It is clear that if $\Delta_{r} f(P)$ exists then $\Delta_{s} f(P), 0 \leqq s \leqq r$, exists. $\Delta^{(k)} f(P)$ shall denote the $k$ th Laplace-Beltrami operator on $f(Q)$ at the point $P, k=0,1, \ldots$ (see [8, p. 212]), and $\Delta_{0}^{(k)} f$ its value when $P$ is the north pole of a system of coordinates with origin at the center of $\Omega$. It is well known that if $Y_{n}(Q)$ is a surface spherical harmonic then $\Delta^{(r)} Y_{n}(Q)=[-n(n+1)]^{r} Y_{n}(Q)$.

2.2. TheOREM. If $Y_{n}(Q), n=0,1, \cdots$, are arbitrary surface spherical harmonics and $P$ is an arbitrary point on $\Omega$ then, for any non-negative integer $r$, $\triangle_{r} Y_{n}(P)$ exists and $\triangle_{r} Y_{n}(P)=\triangle^{(r)} Y_{n}(P)$.

Proof. Since $\Delta_{r}$ and $\Delta^{(r)}$ are linear operators, it is sufficient to show that $\triangle_{r} Y_{n}(P)$ exists and $\triangle_{r} Y_{n}(P)=\Delta^{(r)} Y_{n}(P)$ where $Y_{n}(Q)$ has been normalized so that $Y_{n}(P)=1$. Then $\Delta^{(r)} Y_{n}(P)=\left.\Delta^{(r)} P_{n}([P, Q])\right|_{Q=P}$ (since both are equal to $\left.[-n(n+1)]^{r}\right)$, and by $[7$, p. 298]:

$$
\frac{1}{2 \pi \sin h} \int_{C(P, h)} Y_{n}(Q) d s_{Q}=P_{n}(\cos h)=\frac{1}{2 \pi \sin h} \int_{C(P, h)} P_{n}([P, Q]) d s_{Q}
$$

or $\triangle_{r} Y_{n}(P)=\left.\triangle_{r} P_{n}([P, Q])\right|_{Q=P}$. We therefore need only show that $\left.\triangle_{r} P_{n}([P, Q])\right|_{Q=P}=[-n(n+1)] r$. But by $[6$, p. $21,(18)]$ :

$P_{n}(\cos h)$

$$
=1+\sum_{k=1}^{\infty} \frac{[-n(n+1)][-n(n+1)+1 \cdot 2] \cdots[-n(n+1)+(k-1) k]}{(k !)^{2}} \frac{(1-\cos h)^{k}}{2^{k}}
$$

and the theorem follows easily from (2.1.1) by induction. If $Y_{n}(P)=0$, obviously $\Delta^{(r)} Y_{n}(P)=0=\Delta_{r} Y_{n}(P)$.

III. Statement of main results. 3.1. Definitions AND NOTATION. For an arbitrary series $\sum_{n=0}^{\infty} U_{n}(Q), U_{n}^{(\alpha)}(Q)$ for $\alpha \neq-1,-2, \cdots$, shall denote 
the sum $\sum_{j=0}^{n} \gamma_{n-j}^{(\alpha)} U_{j}(Q)$ where $\gamma_{j}^{(\alpha)} \equiv(\alpha+1) \cdots(\alpha+j) / j$ ! are the ordinary Cesaro coefficients of order $\alpha . \Delta^{(k)} F_{n}$ shall denote the $k$ th difference $k=0,1, \cdots$, of the sequence $\left\{F_{n}\right\}$ where $\Delta^{(1)} F_{n}=F_{n}-F_{n+1} . S[f(Q)]$ shall denote the Laplace series of the function $f(Q)$ defined on $\Omega$ and $\Delta^{(r)} S[f(P)]$ the value at the point $P$ of the series obtained by applying the Laplace-Beltrami operator term by term $r$ times, $r=0,1, \cdots$, to $S[f(Q)]$, i.e., if $S[f(Q)]$ $=\sum_{n=0}^{\infty} Y_{n}(Q)$ then $\Delta^{(r)} S[f(P)]=\sum_{n=0}^{\infty}[-n(n+1)]^{r} Y_{n}(P)$.

DEFINITION. If $Y_{n}(Q), n=0,1, \cdots$, are surface spherical harmonics the anti-Laplace-Beltrami operator of order $r, r=0,1, \cdots$, on $Y_{n}(Q)$, denoted $\Delta^{(-r)} Y_{n}(Q)$, is defined as:

$$
\begin{array}{ll}
\Delta^{(-r)} Y_{0}(Q) \equiv\left\{Y_{0}(Q) \frac{P_{r}[(P, Q)]}{[-r(r+1)]^{r}}\right\}, & \\
\Delta^{(-r)} Y_{n}(Q) \equiv \frac{Y_{n}(Q)}{[-n(n+1)]^{r}}, & n=1,2, \cdots
\end{array}
$$

Obviously $\Delta^{(r)}\left\{\Delta^{(-r)} Y_{n}(Q)\right\}=\Delta^{(-r)}\left\{\Delta^{(r)} Y_{n}(Q)\right\}=Y_{n}(Q)$ for $n$ and $r$ nonnegative integers. Given $f(Q) \in L$ on $\Omega, \Delta^{(-r)} S[f(Q)]$ shall denote the series obtained by applying the anti-Laplace-Beltrami operator term-by-term to $S[f(Q)]$, i.e., if $S[f(Q)]=\sum_{n=0}^{\infty} Y_{n}(Q)$ :

$$
\begin{aligned}
\Delta^{(0)} S[f(Q)]= & \sum_{n=0}^{\infty} Y_{n}(Q), \\
\Delta^{(-r)} S[f(Q)]= & (-1)^{r}\left\{Y_{0}(Q)\left[\frac{P_{r}([P, Q])}{[r(r+1)]^{r}}\right]\right. \\
& \left.+\sum_{n=1}^{\infty} \frac{Y_{n}(Q)}{[n(n+1)]^{r}}\right\}, \quad r=1,2, \cdots .
\end{aligned}
$$

3.2. MAIN THEOREMS. The major results of this paper are embodied in four theorems. The second gives the sufficient conditions for Cesaro summability and follows as a direct consequence of the first. The third yields the necessary conditions and the last combines these results to give the necessary and sufficient conditions.

THEOREM. Let $f(Q)$ be a bounded Borel measurable function on $\Omega$. If $\Delta_{r} f(P)$ exists for some non-negative integer $r$ then $\Delta^{(r)} S[f(P)]$ is $(C-\alpha)$ summable, $\alpha>2 r+1$, to $\Delta_{r} f(P)$.

SUFFICIENCY THEOREM. Let $\sum_{n=0}^{\infty} Y_{n}(Q)$ be a series of surface spherical harmonics on $\Omega$. Let $r$ be a non-negative integer great enough so that $\Delta^{(-r)}\left\{\sum_{n=0}^{\infty} Y_{n}(Q)\right\}$ converges uniformly to $F_{r}(Q)$ on $\Omega$. If $\Delta_{r} F_{r}(P)$ exists then $\sum_{n=0}^{\infty} Y_{n}(Q)$ is $(C-\alpha)$ summable at the point $P, \alpha>2 r+1$, to $\Delta_{r} F_{r}(P)$. 
NeCESSity THEOREM. Let $\sum_{n=0}^{\infty} Y_{n}(Q)$ be a series of surface spherical harmonics on $\Omega$. Let $r$ be a non-negative integer great enough so that $\triangle^{(-r)}\left\{\sum_{n=0}^{\infty} Y_{n}(Q)\right\}$ converges uniformly to $F_{r}(Q)$ on $\Omega$. If $\sum_{n=0}^{\infty} Y_{n}(Q)$ is $(C-\alpha)$ summable, $\alpha$ a non-negative integer, to $s$ at the point $P$ on $\Omega$ then for $r$ an integer greater than $(\alpha+2) / 2, \triangle_{r} F_{r}(P)$ exists and equals $s$.

NECESSITY AND SU:FICIENCY THEOREM. Let $\sum_{n=0}^{\infty} Y_{n}(Q)$ be a series of surface spherical harmonics with $Y_{n}(Q)=O\left(n^{k}\right)$ uniformly on $\Omega$ for some $k$. A necessary and sufficient condition that $\sum_{n=0}^{\infty} Y_{n}(Q)$ be summable $C$ to $s$ at an arbitrary point $P$ on $\Omega$ is that there exist a non-negative integer $r>(k+1) / 2$ such that $\Delta_{r} F_{r}(P)$ exists and equals $s$ where $F_{r}(Q) \equiv \Delta^{(-r)}\left\{\sum_{n=0}^{\infty} Y_{n}(Q)\right\}$.

IV. The sufficiency theorem. In order to facilitate the proof of this theorem we prove the following sublemmas and lemmas:

4.1. Lemma 1. Let $f(Q)$ be a bounded Borel measurable function and $r a$ non-negative integer. If $\triangle_{k} f(P)=0, k=0, \cdots, r$, implies $\Delta^{(r)} S[f(P)]$ is $(C-\alpha)$ summable to zero then $\Delta_{r} f(P)=s$ implies $\Delta^{(r)} S[f(P)]$ is $(C-\alpha)$ summable to $s$.

Proof. Suppose $\Delta_{r} f(P)=s$. We observe first that there exists a finite sum of surface spherical harmonics $T(Q) \equiv \sum_{j=0}^{r} a_{j} P_{j}([P, Q])$ such that $\Delta_{k} T(P)$ $=\triangle_{k} f(P), k=0, \cdots, r$. For, by the theorem of $\$ 2.2$ and the linearity of the operators $\triangle_{k}$ and $\Delta^{(k)}, \Delta_{k} T(Q)=\sum_{j=0}^{r} a_{j} \Delta^{(k)} P_{j}([P, Q])=\Delta^{(k)} T(Q)$, and it is possible to choose the $a_{j}$ so that:

$$
\begin{aligned}
\sum_{j=0}^{r} a_{j} & =\Delta_{0} f(P), \\
\sum_{j=1}^{r}[-j(j+1)]^{k} a_{j} & =\Delta_{k} f(P), \quad k=1,2, \cdots, r .
\end{aligned}
$$

This can clearly be done since the determinant of the above system of $(r+1)$ linear equations is a nonvanishing Vandermonde determinant. Let $F(Q)$ $\equiv f(Q)-T(Q)$. Then $\triangle_{k} F(P)=0, k=0,1, \cdots, r$. Therefore, by hypothesis, $\Delta^{(r)} S[f(P)]$ is $(C-\alpha)$ summable to zero. But since the Laplace series of a surface spherical harmonic is the surface spherical harmonic itself, $\triangle^{(r)} S[F(P)]$ $=\Delta^{(r)} S[f(P)]-\Delta^{(r)} T(P)=\Delta^{(r)} S[f(P)]-s$. The lemma follows immediately.

For the following lemmas and sublemmas we shall assume, unless otherwise stated, that $\alpha$ is non-negative.

\subsection{Sublemma 1.}

$$
\begin{aligned}
& \sum_{j=0}^{n} \frac{\gamma_{n-j}^{(\alpha)}}{\gamma_{n}^{(\alpha)}}[-j(j+1)]^{r}(2 j+1) \sin (j+1 / 2) t \\
& \equiv \sum_{k=0}^{r} a_{k} \sum_{j=0}^{n} \frac{\gamma_{n-j}^{(\alpha)}}{\gamma_{n}^{(\alpha)}} \frac{d^{(2 k+1)}}{d t^{(2 k+1)}} \cos (j+1 / 2) t .
\end{aligned}
$$


Proof. The above identity is a simple application of the following two facts:

$$
\begin{aligned}
&(-1)^{k+1} \frac{d^{(2 k+1)}}{d t^{(2 k+1)}} \cos (j+1 / 2) t=(j+1 / 2)^{2 k+1} \sin (j+1 / 2) t \\
& k=0, \cdots, r .
\end{aligned}
$$

(4.2.3) $[-j(j+1)]^{r}(2 j+1)$ is an odd polynomial of degree $(2 r+1)$ in $(j+1 / 2)$.

Statement (4.2.2) is obvious. Statement (4.2.3) follows easily since

$$
[-j(j+1)]^{r}=\left[(-1)^{r} / 4^{r}\right]\left[(2 j+1)^{2}-1\right]^{r}=(-1)^{r}\left[(j+1 / 2)^{2}-1 / 4\right]^{r} .
$$

4.3. Sublemma 2. For $0<t<2 \pi, k=0, \cdots, r ; r \geqq 0$, and $s>\alpha+2 r+1$ :

$$
\begin{aligned}
& \sum_{j=0}^{n} \frac{\gamma_{n-j}^{(\alpha)}}{\gamma_{n}^{(\alpha)}} \frac{d^{(2 k+1)}}{d t^{(2 k+1)}} \cos (j+1 / 2) t \\
& \equiv \frac{1}{\gamma_{n}^{(\alpha)}} \cdot g\left\{-\sum_{j=1}^{s} \gamma_{n}^{(\alpha-j)} \frac{d^{(2 k+1)}}{d t^{(2 k+1)}}\left[\frac{1}{(2 \sin (t / 2))\left(1-e^{-i t}\right)^{j}}\right]\right. \\
&+\frac{d^{(2 k+1)}}{d t^{(2 k+1)}}\left[\frac{e^{i(n+1) t}}{(2 \sin (t / 2))\left(1-e^{-i t) \alpha}\right.}\right] \\
&\left.-\frac{d^{(2 k+1)}}{d t^{(2 k+1)}}\left[\frac{\sum_{j=n+1}^{\infty} \gamma_{j}^{(\alpha-8-1)} e^{-i(j-n-1) t}}{(2 \sin (t / 2))\left(1-e^{-i t) s}\right.}\right]\right\} .
\end{aligned}
$$

Proof. Since $\sum_{t=0}^{j} \cos (j+1 / 2) t=[\sin (j+1) t] / 2 \sin (t / 2)$ for $0<t<2 \pi$,

$$
\begin{aligned}
& \sum_{j=0}^{n} \frac{d^{(2 k+1)}}{d t^{(2 k+1)}}\left[\gamma_{n-j}^{(\alpha)} / \gamma_{n}^{(\alpha)}\right][\cos (j+1 / 2) t] \\
& \quad=\sum_{j=0}^{n} d^{(2 k+1)} / d t^{(2 k+1)}\left\{\left[\gamma_{n-j}^{(\alpha-1)} / \gamma_{n}^{(\alpha)}\right][\sin (j+1) t] / 2 \sin (t / 2)\right\} \quad \text { for } 0<t<2 \pi .
\end{aligned}
$$

Also,

$$
\begin{aligned}
& \sum_{j=0}^{n}\left[\gamma_{n-j}^{(\alpha-1)} / \gamma_{n}^{(\alpha)}\right][\sin (j+1) t] / 2 \sin (t / 2) \\
& =\left[1 / 2 \gamma_{n}^{(\alpha)} \sin (t / 2)\right] \cdot g\left\{\sum_{j=0}^{n} \gamma_{n-j}^{(\alpha-1)} \exp [i(j+1) t]\right\} \\
& =\left[1 / 2 \gamma_{n}^{(\alpha)} \sin (t / 2)\right] \cdot g\left\{\exp [i(n+1) t] \sum_{j=0}^{n} \gamma_{j}^{(\alpha-1)} \exp (-i j t)\right\},
\end{aligned}
$$


and applying Abel's partial summation formula $s$ times to the expression on the right we obtain (see $[11$, p. 258]):

$$
\begin{aligned}
& \sum_{j=0}^{n} \frac{\stackrel{(\alpha-1)}{\gamma}_{n-j}}{\gamma_{n}^{(\alpha)}} \frac{\sin (j+1) t}{2 \sin (t / 2)} \\
& \quad=\frac{1}{2 \gamma_{n}^{(\alpha)} \sin (t / 2)} \cdot g\left(-\sum_{j=1}^{s} \frac{\gamma_{n}^{(\alpha-j)}}{\left(1-e^{-i t}\right)^{j}}+\frac{e^{i(n+1) t} \sum_{j=0}^{n} \gamma_{j}^{(\alpha-s-1)} e^{-i j t}}{\left(1-e^{-i t}\right)^{s}}\right\} .
\end{aligned}
$$

But since $\alpha-s<-1$ implies $\sum_{j=0}^{\infty}\left|\gamma_{j}^{(\alpha-1-1)}\right|<\infty$, by Abel's limit theorem we have $1 /(1-\exp (-i t))^{\alpha-s}=\sum_{j=0}^{\infty} \gamma_{j}^{(\alpha-s-1)} \exp (-i j t)$. Thus:

$$
\begin{aligned}
& \sum_{j=0}^{n} \frac{\gamma_{n-j}^{(\alpha)}}{\gamma_{n}^{(\alpha)}} \cos (j+1 / 2) t \\
& =\frac{1}{2 \gamma_{n}^{(\alpha)} \sin (t / 2)} \cdot g\left(-\sum_{j=1}^{s} \frac{\gamma_{n}^{(\alpha-j)}}{\left(1-e^{-i t}\right)^{j}}+\frac{e^{i(n+1) t}}{\left(1-e^{-i t}\right)^{\alpha}}-\frac{\sum_{j=n+1}^{\infty} \gamma_{j}^{(\alpha-s-1)} e^{-i(j-n-1) t}}{\left(1-e^{-i t}\right)^{8}}\right\} .
\end{aligned}
$$

Consequently, for $0<t<2 \pi, k=0, \cdots, r ; r \geqq 0$, and $s>\alpha+2 r+1$ :

$$
\begin{aligned}
& \sum_{j=0}^{n} \frac{\gamma_{n-j}^{(\alpha)}}{\gamma_{n}^{(\alpha)}} \frac{d^{(2 k+1)}}{d t^{(2 k+1)}} \cos (j+1 / 2) t \\
& \equiv \frac{1}{\gamma_{n}^{(\alpha)}} \cdot g\left\{-\sum_{j=1}^{s} \gamma_{n}^{(\alpha-j)} \frac{d^{(2 k+1)}}{d t^{(2 k+1)}}\left[\frac{1}{(2 \sin (t / 2))\left(1-e^{-i t}\right)^{j}}\right]\right. \\
& \left.\quad+\frac{d^{(2 k+1)}}{d t^{(2 k+1)}}\left[\frac{e^{i(n+1) t}}{(2 \sin (t / 2))\left(1-e^{-i t}\right)^{\alpha}}\right]-\frac{d^{(2 k+1)}}{d t^{(2 k+1)}}\left[\frac{\sum_{j=n+1}^{\infty} \gamma_{j}^{(\alpha-s-1)} e^{-i(j-n-1) t}}{(2 \sin (t / 2))\left(1-e^{-i t}\right)^{s}}\right]\right\}
\end{aligned}
$$

where the last expression on the right exists since for $s>\alpha+2 r+1$ the absolute value of $\sum_{j=n+1}^{\infty} d^{(2 k+1)} / d t^{(2 k+1)}\left\{\gamma_{j}^{(\alpha-s-1)} \exp [-i(j-n-1) t]\right\}$ is bounded by a constant multiple of the convergent series $\sum_{j=n+1}^{\infty} j^{(\alpha+2 k+1)-(s+1)}$, and consequently converges uniformly for $0<t<2 \pi$.

4.4. Sublemma 3. For $0<t<2 \pi, h \geqq 0$, and $n \geqq 1$ there exist positive constants $K_{1}, K_{2}, K_{3}$ such that:

$$
\left|-\sum_{j=1}^{s} \gamma_{n}^{(\alpha-j)} \frac{d^{h}}{d t^{h}}\left[\frac{1}{(2 \sin (t / 2))\left(1-e^{-i t}\right)^{i}}\right]\right|<K_{1} \sum_{j=1}^{s} \frac{n^{(\alpha-j)}}{t^{(j+h+1)}},
$$

$$
\left|\frac{d^{(h)}}{d t^{(h)}} \frac{e^{i(n+1) t}}{(2 \sin (t / 2))\left(1-e^{-i t}\right)^{\alpha}}\right|<K_{2} \sum_{\mu=0}^{h} \frac{n^{\mu}}{t^{(\alpha+1+h-\mu)}},
$$




$$
\left|\frac{d^{(h)}}{d t^{(h)}} \frac{\sum_{j=n+1}^{\infty} \gamma_{j}^{(\alpha-8-1)} e^{-i(j-n-1) t}}{(2 \sin (t / 2))\left(1-e^{-i t}\right)^{s}}\right|<K_{3} \sum_{\mu=0}^{h} \frac{n^{(\alpha-s+\mu)}}{t^{(8+h+1-\mu)}}
$$

for $s>\alpha+h$.

Proof. We observe that the $h$ th derivative of a product of two functions $A(t)$ and $B(t)$ is given by:

$$
\frac{d^{h}}{d t^{h}} A(t) \cdot B(t)=\sum_{\mu=0}^{h}\left(\begin{array}{l}
h \\
\mu
\end{array}\right) A^{(\mu)}(t) B^{(h-\mu)}(t) .
$$

Since $\mid d^{(v)} / d t^{(v)}[1 / \sin (t / 2)]<C_{1} / t^{v+1}$ and $\left|d^{(v)} / d t^{(v)}\left[1 /\left(1-e^{-i t}\right)^{\gamma}\right]\right|<C_{2} / t^{\gamma+v}$ where $C_{1}, C_{2}>0$, there exists a positive constant $C$ such that:

$$
\left|\frac{d^{(h)}}{d t^{(h)}} \frac{1}{(2 \sin (t / 2))\left(1-e^{-i t}\right)^{\beta}}\right|<\frac{C}{t^{\beta+h+1}} .
$$

Thus from (4.4.4) and (4.4.5) we obtain (4.4.1) and (4.4.2) noticing that for the former inequality $\gamma_{n}^{(k)}=O\left(n^{k}\right)$. With

$$
A(t) \equiv-\sum_{j=n+1}^{\infty} \gamma_{j}^{(\alpha-8-1)} \exp [-i(j-n-1) t]
$$

and $B(t) \equiv 1 /[2 \sin (t / 2)][1-\exp (-i t)]^{i}$ we again employ (4.4.4) and (4.4.5) to obtain (4.4.3), noticing that:

$$
\begin{aligned}
\left|-\frac{d^{(\mu)}}{d t^{(\mu)}} \sum_{j=n+1}^{\infty} \gamma_{j}^{(\alpha-8-1)} e^{-i(j-n-1) t}\right| & <K \sum_{j=n+1}^{\infty} j^{(\alpha-s-1)}(j-n-1)^{\mu} \\
& <K \sum_{j=n+1}^{\infty} j^{(\alpha+\mu-s-1)}<K n^{(\alpha+\mu-s)}
\end{aligned}
$$

for $\mu=0, \cdots, h$ and $s>\alpha+h$ where $K>0$.

4.5. Sublemma 4. For $\beta>1$ and $0<\theta<\pi / 2$ there exists a positive constant $K$ such that:

$$
\int_{\theta}^{\pi} \frac{d t}{t^{\beta}(\cos \theta-\cos t)^{1 / 2}}<\frac{K}{\theta \beta}
$$
that:

Proof. Using the identity $\cos \theta-\cos t=2 \sin [(t+\theta) / 2] \sin [(t-\theta) / 2]$ we see

$$
\int_{\theta}^{\pi} \frac{d t}{t^{\beta}(\cos \theta-\cos t)^{1 / 2}}=\frac{1}{2^{1 / 2}} \int_{\theta}^{\pi} \frac{d t}{\left.t^{\beta}[\sin ((t+\theta) / 2) \sin ((t-\theta) / 2))\right]^{1 / 2}} .
$$


But for $0<\theta \leqq t \leqq \pi, \quad \sin [(t+\theta) / 2]=\sin (t / 2) \cos (\theta / 2)+\cos (t / 2) \sin (\theta / 2)$ $\geqq \sin (\theta / 2) \cos (\theta / 2)+\cos (t / 2) \sin (\theta / 2) \geqq \sin (\theta / 2) \cos (\theta / 2)=\sin \theta / 2$ and hence:

$$
\begin{array}{r}
\frac{1}{2^{1 / 2}} \int_{\theta}^{\pi} \frac{d t}{t^{\beta}[\sin ((t+\theta) / 2) \sin ((t-\theta) / 2)]^{1 / 2}} \leqq \frac{1}{(\sin \theta)^{1 / 2}} \int_{\theta}^{\pi} \frac{d t}{t^{\beta}[\sin ((t-\theta) / 2)]^{1 / 2}} \\
\text { for } 0<\theta<\pi .
\end{array}
$$

Also, for $0<\theta \leqq t \leqq \pi, \sin [(t-\theta) / 2] \geqq[2 / \pi][(t-\theta) / 2]$ since $(t-\theta) / 2<\pi / 2$. Thus:

$$
\frac{1}{(\sin \theta)^{1 / 2}} \int_{\theta}^{\pi} \frac{d t}{t^{\beta}[\sin ((t-\theta) / 2)]^{1 / 2}} \leqq \frac{\pi^{1 / 2}}{(\sin \theta)^{1 / 2}} \int_{\theta}^{\pi} \frac{d t}{t^{\beta}(t-\theta)^{1 / 2}} \quad \text { for } 0<\theta<\pi .
$$

Obviously $\int_{\theta}^{\pi} 1 / t^{\beta}(t-\theta)^{1 / 2} d t<\int_{\theta}^{\infty} 1 / t^{\beta}(t-\theta)^{1 / 2} d t$ and dividing the integral on the right into two parts,

$$
\int_{\theta}^{\infty} 1 / t^{\beta}(t-\theta)^{1 / 2} d t=\int_{\theta}^{2 \theta} 1 / t^{\beta}(t-\theta)^{1 / 2} d t+\int_{2 \theta}^{\infty} 1 / t^{\beta}(t-\theta)^{1 / 2} d t .
$$

Noticing that

$$
\int_{\theta}^{2 \theta} 1 / t^{\beta}(t-\theta)^{1 / 2} d t \leqq\left[1 / \theta^{\beta}\right] \int_{\theta}^{2 \theta} 1 /(t-\theta)^{1 / 2} d t=2 / \theta^{(\beta-1 / 2)}
$$

and

$$
\int_{2 \theta}^{\infty} 1 / t^{\beta}(t-\theta)^{1 / 2} d t \leqq\left[1 / \theta^{1 / 2}\right] \int_{2 \theta}^{\infty} 1 / t^{\beta} d t=\left[1 / 2^{(\beta-1)}(\gamma-1)\right]\left[1 / \theta^{(\beta-1 / 2)}\right]
$$

for $\beta>1$,

we see that there exists a constant $C>0$ such that:

$$
\int_{\theta}^{\pi} \frac{d t}{t^{\beta}(\cos \theta-\cos t)^{1 / 2}}<\frac{C}{\theta^{(\beta-1 / 2)}(\sin \theta)^{1 / 2}} \quad \text { for } \beta>1,0<\theta<\pi .
$$

But for $0<\theta<\pi / 2, \sin \theta \geqq(2 / \pi) \theta$ and consequently (4.5.1) follows.

4.6. Sublemma 5. For $0<\theta<\pi / 2$ there exist positive constants $K_{1}, K_{2}, K_{3}$ such that:

$$
\frac{2^{1 / 2}}{\pi} \int_{\theta}^{\pi} \frac{G(n, t, \alpha)}{(\cos \theta-\cos t)^{1 / 2}} d t<K_{1} \sum_{j=1}^{\dot{ }} \frac{n^{-j}}{\theta^{(j+h+1)}}
$$

where 


$$
\begin{gathered}
G(n, t, \alpha) \equiv \frac{1}{\gamma_{n}^{(\alpha)}} \sum_{j=1}^{s} \frac{n^{(\alpha-j)}}{t^{(j+h+1)}}, \quad h=1,3, \cdots, 2 r+1, \\
\frac{2^{1 / 2}}{\pi} \int_{\theta}^{\pi} \frac{H(n, t, \alpha)}{(\cos \theta-\cos t)^{1 / 2}} d t<K_{2} \frac{n^{(h-\alpha)}}{\theta^{(\alpha+1)}}
\end{gathered}
$$

where

$$
\begin{gathered}
H(n, t, \alpha) \equiv \frac{1}{\gamma_{n}^{(\alpha)}} \cdot \frac{n^{h}}{t^{(\alpha+1)}}, \quad h=1,3, \cdots, 2 r+1, \\
\frac{2^{1 / 2}}{\pi} \int_{0}^{\pi} \frac{L(n, t, \alpha)}{(\cos \theta-\cos t)^{1 / 2}} d t<K_{3} \frac{n^{(-s+h)}}{\theta^{s+1}}
\end{gathered}
$$

where

$$
L(n, t, \alpha) \equiv \frac{1}{\gamma_{n}^{(\alpha)}} \cdot \frac{n^{(\alpha-s+h)}}{t^{s+1}}, \quad h=1,3, \cdots, 2 r+1 .
$$

Proof. These results follow immediately from 4.5 Sublemma 4 and the fact that $\gamma_{n}^{(\alpha)} \sim n^{\alpha} / \Gamma(\alpha+1)$ for $\alpha \neq-1,-2, \cdots$.

4.7. Sublemma 6. For $s>\alpha+h, h=1,3, \cdots, 2 r+1, \alpha>2 r+1$, and $n$ a positive integer, there exists a positive constant $K$ such that:

$$
\begin{gathered}
\int_{1 / n}^{\pi} \sum_{j=1}^{s} \frac{n^{-j}}{\theta^{(j+h+1)}} \sin ^{(2 r+1)} \theta d \theta<K, \\
\int_{1 / n}^{\pi} \frac{n^{(h-\alpha)}}{\theta^{(\alpha+1)}} \sin ^{(2 r+1)} \theta d \theta<K, \\
\int_{1 / n}^{\pi} \frac{n^{(-s+h)}}{\theta^{(s+1)}} \sin ^{(2 r+1)} \theta d \theta<K .
\end{gathered}
$$

Proof. For $h=1,3, \cdots, 2 r+1$ :

$$
\int_{1 / n}^{\pi} \sum_{j=1}^{s} \frac{n^{-j}}{\theta^{(j+h+1)}} \sin ^{(2 r+1)} \theta d \theta \leqq \int_{1 / n}^{\pi} \sum_{j=1}^{\infty} \frac{n^{-j}}{\theta^{(j+2 r+2)}} \sin ^{(2 r+1)} \theta d \theta,
$$

and since $\sin \theta / \theta \leqq 1$ for $0<\theta \leqq \pi$ :

$$
\int_{1 / n}^{\pi} \sum_{j=1}^{s} \frac{n^{-j}}{\theta^{(j+2 r+2)}} \sin ^{(2 r+1)} \theta d \theta \leqq \int_{1 / n}^{\pi} \sum_{j=1}^{s} \frac{n^{-j}}{\theta^{(j+1)}} d \theta .
$$

Integrating the expression on the right we obtain:

$$
\int_{1 / n}^{\pi} \sum_{j=1}^{\infty} \frac{n^{-j}}{\theta^{(j+1)}} d \theta=\sum_{j=1}^{s} \frac{1}{j}\left[1-\frac{1}{(\pi n)^{i}}\right]<K
$$


where $K$ is a positive constant, and (4.7.1) follows. Similarly we find that for $h=1,3, \cdots, 2 r+1$ and $\alpha>2 r+1$ :

$$
\begin{aligned}
\int_{1 / n}^{\pi} \frac{n^{(h-\alpha)}}{\theta^{(\alpha+1)}} \sin ^{(2 r+1)} \theta d \theta & \leqq n^{(2 r+1-\alpha)} \int_{1 / n}^{\pi} \frac{\sin ^{(2 r+1)}}{\theta^{(\alpha+1)}} d \theta \leqq n^{(2 r+1-\alpha)} \int_{1 / n}^{1} \frac{d \theta}{\theta^{(\alpha-2 r)}} \\
& =\frac{1}{\alpha-(2 r+1)}\left[1-\frac{1}{(\pi n)^{\alpha-(2 r+1)}}\right] .
\end{aligned}
$$

Thus for $\alpha>2 r+1$ we see that with $K=1 /[\alpha-(2 r+1)]>0$ (4.7.2) holds. Finally, for $h=1,3, \cdots, 2 r+1$ :

$$
\begin{aligned}
& \int_{1 / n}^{\pi} \frac{n^{(-8+h)}}{\theta^{(8+1)}} \sin ^{(2 r+1)} \theta d \theta \leqq n^{(-s+2 r+1)} \int_{1 / n}^{\pi} \frac{d \theta}{s-2 r} \\
& \leqq \frac{1}{s-(2 r+1)}\left[1-\frac{1}{(\pi n)^{s-(2 r+1)}}\right] \text {, }
\end{aligned}
$$

and therefore, for $s>\alpha+h$, we have (4.7.3) with $K=1 /[s-(2 r+1)]>0$.

The following notation will be used in the remaining lemmas and theorems of this section: We let $K_{n}^{(\alpha, r)}(\cos \theta)$ denote the $n$th $(C-\alpha)$ partial sum of

$$
\sum_{j=0}^{\infty}[-j(j+1)]^{r}(2 j+1) P_{j}(\cos \theta)
$$

i.e.,

$$
K_{n}^{(\alpha, r)}(\cos \theta) \equiv \sum_{j=0}^{n}\left[\gamma_{n-j}^{(\alpha)} / \gamma_{n}^{(\alpha)}\right][-j(j+1)]^{r}(2 j+1) P_{j}(\cos \theta)
$$

4.8. LEMмA 2. Let $1 / n \leqq \eta \leqq \pi / 2$ where $n$ is a positive integer. For $\alpha>2 r+1$ there exists a positive constant $K$ independent of $\eta$ such that:

$$
\int_{1 / n}^{\eta}\left|K_{n}^{(\alpha, r)}(\cos \theta)\right| \sin \theta^{(2 r+1)} d \theta<K .
$$

Proof. From Mehler's integral representation of the Legendre polynomials $[6$, p. $27,(27)]$ :

$$
P_{j}(\cos \theta)=\frac{2^{1 / 2}}{\pi} \int_{\theta}^{\pi} \frac{\sin (j+1 / 2) t}{(\cos \theta-\cos t)^{1 / 2}} d t \quad \text { for } 0<\theta<\pi ; j=0,1, \cdots
$$

we see that for $0<\theta<\pi$ :

$$
K_{n}^{(\alpha, r)}(\cos \theta)=\frac{2^{1 / 2}}{\pi} \int_{\theta}^{\pi} \frac{\sum_{j=0}^{n} \frac{\gamma_{n-j}^{(\alpha)}}{\gamma_{n}^{(\alpha)}}[-j(j+1)] r(2 j+1) \sin (j+1 / 2) t}{(\cos \theta-\cos t)^{1 / 2}} d t
$$


and thus by 4.2 Sublemma 1 , for $0<\theta<\pi$ :

$$
K_{n}^{(\alpha, r)}(\cos \theta)=\frac{2^{1 / 2}}{\pi} \int_{\theta}^{\pi} \frac{\sum_{k=0}^{r} a_{k} \sum_{j=0}^{n} \frac{\gamma_{n-j}^{(\alpha)}}{\gamma_{n}^{(\alpha)}} \frac{d^{(2 k+1)}}{d t^{(2 k+1)}} \cos (j+1 / 2) t}{(\cos \theta-\cos t)^{1 / 2}} d t .
$$

Utilizing the fact that $|g\{f(z)\}| \leqq|f(z)|$ we have, by 4.3 Sublemma 2 and 4.4 Sublemma 3 , for $0<t<\pi, k=0, \cdots, r$, and $r \geqq 0$, the existence of a constant $K>0$ such that:

$$
\begin{aligned}
\mid \sum_{j=0}^{n} \frac{\gamma_{n-j}^{(\alpha)}}{\gamma_{n}^{(\alpha)}} \frac{d^{(2 k+1)}}{d t^{(2 k+1)}} & \cos (j+1 / 2) t \mid \\
& <\frac{K}{\gamma_{n}^{(\alpha)}}\left\{\sum_{j=1}^{s} \frac{n^{(\alpha-j)}}{t^{(j+2 k+2)}}+\sum_{\mu=0}^{2 k+1} \frac{n^{\mu}}{t^{(\alpha+2 k+2-\mu)}}+\sum_{\mu=0}^{2 k+1} \frac{n^{(\alpha-s+\mu)}}{t^{(s+2 k+1-\mu)}}\right\}
\end{aligned}
$$

where $s$ is fixed so that $s>\alpha+2 r+1$. For $1 / n \leqq t$, noticing that there exists a constant $K>0$ such that

$$
\sum_{\mu=0}^{2 k+1} n^{\mu} / t^{(\alpha+2 k+2-\mu)}<K n^{2 k+1} / t^{\alpha+1}
$$

and

$$
\sum_{\mu=0}^{2 k+1} n^{(\alpha-s+\mu)} / t^{(s+2 k+1-\mu)}<K n^{(\alpha-s+2 k+1)} / t^{s+1}
$$

for $k=0, \cdots, r$, we see that with $1 / n \leqq t<\pi$ there exists a $K>0$ such that:

$$
\begin{aligned}
& \left|\sum_{j=0}^{n} \frac{\gamma_{n-j}^{(\alpha)}}{\gamma_{n}^{(\alpha)}} \frac{d^{(2 k+1)}}{d t^{(2 k+1)}} \cos (j+1 / 2) t\right| \\
& \quad<\frac{K}{\gamma_{n}^{(\alpha)}}\left\{\sum_{j=1}^{s} \frac{n^{(\alpha-j)}}{t^{(j+2 k+2)}}+\frac{n^{2 k+1}}{t^{\alpha+1}} \frac{n^{(\alpha-s+2 k+1)}}{t^{s+1}}\right\}, \quad k=0, \cdots, r .
\end{aligned}
$$

Thus by 4.6 Sublemma 5 , for $0<1 / n \leqq \theta \leqq \pi / 2$ there exists a constant $K>0$ such that:

$$
\begin{aligned}
\frac{2^{1 / 2}}{\pi} \int_{\theta}^{\pi} \frac{\left|\sum_{j=0}^{n} \frac{\gamma_{n-j}^{(\alpha)}}{\gamma_{n}^{(\alpha)}} \frac{d^{(2 k+1)}}{d t^{(2 k+1)}} \cos (j+1 / 2) t\right|}{(\cos \theta-\cos t)^{1 / 2}} d t \\
\quad<K\left\{\sum_{j=1}^{s} \frac{n^{(-j)}}{\theta^{(j+2 k+2)}} \frac{n^{(2 k+1-\alpha)}}{\theta^{(\alpha+1)}}+\frac{n^{(-s+2 k+1)}}{\theta^{(\bullet+1)}}\right\}, \quad k=0, \cdots, r .
\end{aligned}
$$


Therefore from (4.8.3) and (4.8.4) we see that, for $0<1 / n \leqq \theta \leqq \pi / 2$, there exists a constant $K>0$ such that:

$$
\left|K_{n}^{(\alpha, r)}(\cos \theta)\right|<K\left\{\sum_{k=0}^{r} \sum_{j=1}^{s} \frac{n^{(-j)}}{\theta^{(j+2 k+2)}}+\sum_{k=0}^{r} \frac{n^{(2 k+1-\alpha)}}{\theta^{(\alpha+1)}}+\sum_{k=0}^{r} \frac{n^{(-s+2 k+1)}}{\theta^{(s+1)}}\right\} .
$$

Employing 4.7 Sublemma 6 and noticing that the integrands in (4.7.1), (4.7.2), and (4.7.3) are positive for the domain of integration $[1 / n, \eta], \eta \leqq 2$, the result follows.

4.9. Lemma 3. Let $f(\theta, \phi)$ be a bounded measurable function on $\Omega, r$ a nonnegative integer, and $\eta$ any positive number less than $\pi$. If $\alpha>2 r+1$ then:

(4.9.1) $[1 / 4 \pi] \iint_{\Omega-D(P, \eta)} f(M) K_{n}^{(\alpha, r)}([P, M]) d \Omega_{M} \rightarrow 0$ as $n \rightarrow \infty$.

Proof. From Lemma 5 of $\left[8\right.$, p. 217] we have: If $0<h_{1}<h_{2}<\pi$ and $F_{1}(x)$ is a bounded measurable function on $\Omega$ which is equal to zero in $D\left(x_{0}, h_{2}\right)$ then for $x$ in $D\left(x_{0}, h_{1}\right) \Delta^{(r)} S\left[F_{1}(x)\right]$ is uniformly summable $(C-\alpha)$ to zero, $\alpha>2 r+1$.

Define $F_{1}(Q)$ by:

$$
\begin{aligned}
F_{1}(Q) & \equiv 0 \quad \text { in } \quad D(P, \eta) \\
& \equiv f(Q) \quad \text { in } \quad \Omega-D(P, \eta) .
\end{aligned}
$$

Then by the above mentioned lemma we have $\Delta^{(r)} S\left[F_{1}(P)\right]$ summable $(C-\alpha)$ to zero $\alpha>2 r+1$. But $\Delta^{(r)} S\left[F_{1}(P)\right]=[1 / 4 \pi] \iint_{\Omega} F_{1}(M) K_{n}^{(\alpha, r)}([P, M]) d \Omega_{M}$ and by (4.9.2),

$$
\triangle^{(r)} S\left[F_{1}(P)\right]=1 / 4 \pi \iint_{\Omega-D(P, \eta)} f(M) K_{n}^{(\alpha, r)}([P, M]) d \Omega_{M}
$$

4.10. LemMa 4. For $r$ a non-negative integer and $n$ a positive integer, there exists a constant $K>0$ such that:

$$
\int_{0}^{1 / n}\left|K_{n}^{(\alpha, r)}(\cos \theta)\right| \sin ^{(2 r+1)} \theta d \theta<K
$$

Proof. Since $\left|P_{j}(\cos \theta)\right| \leqq 1, j=0,1, \cdots$,

$$
\left|K_{n}^{(\alpha, r)}(\cos \theta)\right| \leqq \sum_{j=0}^{n}\left[\gamma_{n-j}^{(\alpha)} / \gamma_{n}^{(\alpha)}\right][j(j+1)]^{r}[2 j+1]
$$

Consequently there exists a constant $K>0$ such that 


$$
\left|K_{n}^{(\alpha, r)}(\cos \theta)\right|<K n^{2 r+1} \sum_{j=0}^{n} \gamma_{n-j}^{(\alpha)} / \gamma_{n}^{(\alpha)} \leqq K n^{2 r+1}\left[\gamma_{n}^{(\alpha+1)} / \gamma_{n}^{(\alpha)}\right]
$$

But since $\lim _{n \rightarrow \infty}\left[\gamma_{n}^{(\alpha)} / n^{\alpha}\right]=1 / \Gamma(\alpha+1)$ for $\alpha \neq-1,-2, \cdots,\left|K_{n}^{(\alpha, r)}(\cos \theta)\right|$ $<K n^{2(r+1)}$ and (4.10.1) follows.

4.11. Theorem. Let $f(Q)$ be a bounded Borel measurable function on $\Omega$. If $\triangle_{r} f(P)$ exists for some non-negative integer $r$ then $\Delta^{(r)} S[f(P)]$ is $(C-\alpha)$ summable, $\alpha>2 r+1$, to $\Delta_{r} f(P)$.

Proof. Without loss of generality it may be assumed that $P$ is the north pole of a system of spherical coordinates whose origin is at the center of $\Omega$. Furthermore by 4.1 Lemma 1 it may be assumed that $\Delta_{k} f(P)=0, k=0, \cdots, r$, and it remains to show that $\Delta^{(r)} S[f(P)]$ is $(C-\alpha)$ summable, $\alpha>2 r+1$, to zero. We have $S[f(P)]=[1 / 4 \pi] \sum_{j=0}^{\infty}(2 j+1) \iint_{\Omega} f(M) P_{j}([M, P]) d \Omega_{M}$ and $\Delta^{(r)} S[f(P)]=[1 / 4 \pi] \sum_{j=0}^{\infty} \iint_{\Omega}[-j(j+1)]^{r}(2 j+1) f(M) P_{j}([M, P]) d \Omega_{M}$. Denoting the $(C-\alpha)$ partial sums of this latter series by $C_{n}^{(\alpha, r)}(P)$, clearly

$$
C_{n}^{(\alpha, r)}(P)=[1 / 4 \pi] \iint_{\Omega} f(M) K_{n}^{(\alpha, r)}([M, P]) d \Omega_{M} .
$$

Since $\Delta_{k} f(P)=0, k=0, \cdots, r$, given an arbitrary $\epsilon>0$ there exists a $\delta(\epsilon / 2 K)$ $>0$ such that:

$$
\left|\frac{(1 / 2 \pi \sin h) \int_{C(P, \theta)} f(Q) d s_{Q}}{\sin ^{2 r} \theta}\right|<\frac{\epsilon}{2 K}
$$

whenever $\theta<\delta(\epsilon / 2 K)$ where $K$ is chosen such that $K=\max \left(K_{1}, K_{2}\right)$ and $K_{1}$, $K_{2}$ are the constants of 4.8 Lemma 2 and 4.10 Lemma 4 respectively. Let $D(P, \eta)$ be the spherical cap of radius $\eta$ about $P$ with $\eta$ chosen such that $\eta=\min (\delta(\epsilon / 2 K), \pi / 2)$ and let $n$ be a positive integer chosen so that $1 / n \leqq \eta$.

Then since:

$$
\begin{aligned}
\left|C_{n}^{(\alpha, r)}(P)\right|= & {[1 / 4 \pi] \mid \int_{0}^{\eta} \int_{0}^{2 \pi} f(\theta, \phi) K_{n}^{(\alpha, r)}(\cos \theta) \sin \theta d \phi d \theta } \\
& +\iint_{\Omega-D(P, \eta)} f(M) K_{n}^{(\alpha, r)}([P, M]) d \Omega_{M} \mid \\
\left|C_{n}^{(\alpha, r)}(P)\right|<[\epsilon / 2 K] \int_{0}^{\eta}\left|K_{n}^{(\alpha, r)}(\cos \theta)\right| \sin ^{(2 r+1)} \theta d \theta & \\
& +[1 / 4 \pi]\left|\iint_{\Omega-D(P, \eta)} f(M) K_{n}^{(\alpha, r)}([P, M]) d \Omega_{M}\right|
\end{aligned}
$$

by (4.11.1). Dividing the first integral on the right into two parts it follows that: 


$$
\begin{aligned}
\left|C_{n}^{(\alpha, r)}(P)\right|< & {[\epsilon / 2 K] \int_{0}^{1 / n}\left|K_{n}^{(\alpha, r)}(\cos \theta)\right| \sin ^{(2 r+1)} \theta d \theta } \\
& +[\epsilon / 2 K] \int_{1 / n}^{\eta}\left|K_{n}^{(\alpha, r)}(\cos \theta)\right| \sin ^{(2 r+1)} \theta d \theta \\
& +[1 / 4 \pi]\left|\iint_{\Omega-D(P, \eta)} f(M) K_{n}^{(\alpha, r)}([P, M]) d \Omega_{M}\right| .
\end{aligned}
$$

Thus by 4.8 Lemma 2 and 4.10 Lemma 4 , for $\alpha>2 r+1$ :

$$
\left|C_{n}^{(\alpha, r)}(P)\right|<\epsilon+[1 / 4 \pi]\left|\iint_{\Omega-D(P, \eta)} f(M) K_{n}^{(\alpha, r)}([P, M]) d \Omega_{M}\right|
$$

and therefore by 4.9 Lemma $3, \lim \sup _{n \rightarrow \infty} C_{n}^{(\alpha, r)}(P) \leqq \epsilon$. Since $\epsilon>0$ was chosen arbitrarily, the theorem follows.

4.12. SUFFICIENCY THEOREM. Let $\sum_{n=0}^{\infty} Y_{n}(Q)$ be a series of surface spherical harmonics on $\Omega$. Let $r$ be a non-negative integer great enough so that $\Delta^{(-r)}\left\{\sum_{n=0}^{\infty} Y_{n}(Q)\right\}$ converges uniformly to $F_{r}(Q)$ on $\Omega$. If $\Delta_{r} F_{r}(P)$ exists then $\sum_{n=0}^{\infty} Y_{n}(Q)$ is $(C-\alpha)$ summable at the point $P, \alpha>2 r+1$, to $\Delta_{r} F_{r}(P)$.

Proof. The theorem follows immediately from the preceding one after noticing that $\Delta^{(-r)}\left\{\sum_{n=0}^{\infty} Y_{n}(Q)\right\}$ converges uniformly on $\Omega$ to a continuous function $F_{r}(Q)$, and that $\Delta^{(r)} S\left[F_{r}(P)\right]=\sum_{n=0}^{\infty} Y_{n}(P)$.

V. The necessary conditions for $C$ summability. The following three lemmas facilitate the proof of the main theorem of this section:

5.1. Lemma. Let $Y_{n}(Q), n=1,2, \cdots$, be surface spherical harmonics such that $\sum_{n=1}^{\infty} Y_{n}(P)$ is $(C-\alpha)$ summable, $\alpha$ a non-negative integer, for $P$ an arbitrary point on $\Omega$. Then for $r>(\alpha+1) / 2$ :

$$
(-1)^{r} \sum_{n=1}^{\infty} Y_{n}^{(\alpha)}(P) \Delta^{(\alpha+1)}\left\{\frac{P_{n}(\cos h)}{[n(n+1)]^{r}}\right\} \equiv \sum_{k=0}^{r} a_{k} \frac{(1-\cos h)^{k}}{2^{k}}+L^{r}(\cos h)
$$

where

$$
\begin{aligned}
& a_{0}=\text { the }(C-\alpha) \text { sum of the series }(-1)^{r} \sum_{n=1}^{\infty} Y_{n}(P) \cdot \frac{1}{[n(n+1)]^{r}}, \\
& a_{k}=\text { the }(C-\alpha) \text { sum of the series } \\
& \qquad(-1)^{r} \sum_{n=1}^{\infty} Y_{n}(P) \frac{\Delta_{0}\left(\Delta_{0}+1 \cdot 2\right) \cdots\left[\Delta_{0}+(k-1) k\right] P_{n}}{[n(n+1)]^{r}}
\end{aligned}
$$

$k=1,2, \cdots, r$, and $L^{r}(\cos h)$ is a convergent series.

Proof. From $[1$, p. $21,(8)]$ we know that if $\left\{f_{n}\right\}$ and $\left\{g_{n}\right\}$ are two sequences then: 


$$
\Delta^{(j)}\left[f_{n} \cdot g_{n}\right]=\sum_{k=0}^{j}\left(\begin{array}{l}
j \\
k
\end{array}\right) \Delta^{(k)} f_{n} \cdot \Delta^{(j-k)} g_{n+k} .
$$

Letting $f_{n} \equiv P_{n}(\cos h), g_{n} \equiv 1 /[n(n+1)] r$, and noting that

$$
\Delta^{(k)}\left[1 / n^{s}\right]=O\left(1 / n^{s+k}\right) \quad \text { for } k \geqq 0,\left|P_{n}(\cos h)\right| \leqq 1,
$$

and $Y_{n}^{(\alpha)}(P)=O\left(n^{\alpha}\right)$, we see that for $r>(\alpha+1) / 2$ :

$$
\sum_{n=1}^{\infty}\left|Y_{n}^{(\alpha)}(P)\right|\left|\Delta^{(\alpha+1)}\left\{\frac{P_{n}(\cos h)}{n(n+1)^{r}}\right\}\right|<K \sum_{n=1}^{\infty} \frac{1}{n^{(2 r-\alpha)}}<\infty .
$$

Now from [1, p. 19], if $\left\{f_{n}\right\}$ is an arbitrary sequence then:

$$
\Delta^{(\alpha)} f_{n}=\sum_{j=0}^{\alpha}(-1)^{i}\left(\begin{array}{l}
\alpha \\
j
\end{array}\right) f_{n+j} .
$$

Consequently:

$$
\begin{aligned}
& (-1)^{r} \sum_{n=1}^{\infty} Y_{n}^{(\alpha)}(P) \Delta^{(\alpha+1)}\left\{\frac{P_{n}(\cos h)}{[n(n+1)]^{r}}\right\} \\
& =(-1)^{r} \sum_{n=1}^{\infty} Y_{n}^{(\alpha)}(P)\left\{\sum_{j=0}^{\alpha+1}(-1)^{j}\left(\begin{array}{c}
\alpha+1 \\
j
\end{array}\right) \frac{P_{n+j}(\cos h)}{[(n+j)(n+j+1)]^{r}}\right\},
\end{aligned}
$$

and therefore by (2.2.1):

$$
\begin{aligned}
& (-1)^{r} \sum_{n=1}^{\infty} Y_{n}^{(\alpha)}(P) \Delta^{(\alpha+1)}\left\{\frac{P_{n}(\cos h)}{[n(n+1)]^{r}}\right\} \\
= & (-1)^{r} \sum_{n=1}^{\infty} \sum_{k=0}^{\infty} Y_{n}^{(\alpha)}(P)\left\{\sum_{j=0}^{\alpha+1} \frac{(-1)^{i}\left(\begin{array}{c}
\alpha+1 \\
j
\end{array}\right)}{[(n+j)(n+j+1)]^{r}} C_{n+j, k}\right\} \frac{(1-\cos h)^{k}}{2^{k}}
\end{aligned}
$$

where $C_{n+j, k}$ is defined by:

$$
C_{n+j, 0} \equiv 1 \text {, }
$$

(5.1.8) $\quad C_{n+j, k} \equiv \frac{\Delta_{0}\left(\Delta_{0}+1 \cdot 2\right) \cdots\left[\Delta_{0}+(k-1) k\right] P_{n+j}}{(k !)^{2}}$, for $k \leqq n+j$,

$$
C_{n+j, k} \equiv 0, \quad \text { for } k \geqq n+j+1 .
$$

Thus with $a_{n k}$ defined by:

(5.1.9) $\quad a_{n k} \equiv Y_{n}^{(\alpha)}(P) \sum_{j=0}^{\alpha+1} \frac{(-1)^{i}\left(\begin{array}{c}\alpha+1 \\ j\end{array}\right)}{[(n+j)(n+j+1)]^{r}} C_{n+j, k} \frac{(1-\cos h)^{k}}{2^{k}}$

we have: 


$$
(-1)^{r} \sum_{n=1}^{\infty} Y_{n}^{(\alpha)}(P) \Delta^{(\alpha+1)}\left\{\frac{P_{n}(\cos h)}{[n(n+1)]}\right\}=(-1)^{r} \sum_{n=1}^{\infty} \sum_{k=0}^{\infty} a_{n k} .
$$

It will now be shown that:

$$
\sum_{n=1}^{\infty} \sum_{k=0}^{\infty} a_{n k}=\sum_{k=0}^{r} \sum_{n=1}^{\infty} a_{n k}+\sum_{n=1}^{\infty} \sum_{k=r+1}^{\infty} a_{n k}
$$

where $L^{r}(\cos h) \equiv \sum_{n=1}^{\infty} \sum_{k=r+1}^{\infty} a_{n k}$ is a convergent series. To do this it need only be shown that $\sum_{k=0}^{r} \sum_{n=1}^{\infty} a_{n k}$ is absolutely convergent for, from the convergence of $\sum_{n=1}^{\infty} \sum_{k=0}^{r} a_{n k}$ and $\sum_{n=1}^{\infty} \sum_{k=0}^{\infty} a_{n k}$ (see (5.1.4)), the result is immediate. From (5.1.5) and (5.1.9),

$$
\sum_{n=1}^{\infty}\left|a_{n k}\right|=\sum_{n=1}^{\infty}\left|Y_{n}^{(\alpha)}(P)\right|\left|\Delta^{(\alpha+1)}\left\{C_{n k} /[n(n+1)] r\right\}\left[(1-\cos h)^{k} / 2^{k}\right]\right|,
$$

and thus from (5.1.8) we have:

$$
\begin{aligned}
& \sum_{n=1}^{\infty}\left|a_{n 0}\right| \\
& \quad=\sum_{n=1}^{\infty}\left|Y_{n}^{(\alpha)}(P)\right|\left|\Delta^{(\alpha+1)}\left\{\frac{\Delta_{0}\left(\Delta_{0}+1 \cdot 2\right) \cdots\left[\Delta_{0}+(k-1) k\right] P_{n}}{[n(n+1)]^{r}}\right\}\right|, \\
& \begin{array}{l}
\sum_{n=1}^{\infty}\left|a_{n k}\right| \\
\quad=\frac{1}{(k !)^{2}} \sum_{n=1}^{\infty}\left|Y_{n}^{(\alpha)}(P)\right| \mid \Delta^{(\alpha+1)}\left\{\frac{\Delta_{0}\left(\Delta_{0}+1 \cdot 2\right) \cdots\left[\Delta_{0}+(k-1) k\right] P_{n}}{[n(n+1)]^{r}}\right\} \\
\cdot \frac{(1-\cos h)^{k}}{2^{k}} \mid, \quad k=1, \cdots, r .
\end{array}
\end{aligned}
$$

The highest degree of the polynomial $\Delta_{0}\left(\Delta_{0}+1 \cdot 2\right) \cdots\left[\Delta_{0}+(k-1) k\right] P_{n}$ $\equiv[-n(n+1)][-n(n+1)+1 \cdot 2] \cdots[-n(n+1)+(k-1) k]$ for $k=1, \cdots, r$, is obviously $2 r$. Thus since $\Delta^{(\alpha+1)}(-1)^{r}=0$ for $\alpha \geqq 0$,

$$
\begin{aligned}
\left|\Delta^{(\alpha+1)} \frac{\Delta_{0}\left(\Delta_{0}+1 \cdot 2\right) \cdots\left[\Delta_{0}+(k-1) k\right] F_{n}}{[n(n+1)]^{r}}\right| & <K \Delta^{(\alpha+1)}\left\{\frac{1}{[n(n+1)]^{r}}\right\} \\
& <\frac{K}{n^{(\alpha+3)}} \quad \text { for } k=1, \cdots, r .
\end{aligned}
$$

Also, $\Delta^{(\alpha+1)}\{1 /[n(n+1)] r\}=O\left(1 / n^{2(\alpha+1)}\right)$ for $r>(\alpha+1) / 2$. Thus since $Y_{n}^{(\alpha)}(P)=O\left(n^{\alpha}\right)$, from (5.1.12) we see that $\sum_{n=1}^{\infty} a_{n k}, k=0, \cdots, r$, converges absolutely. Therefore from (5.1.7), (5.1.8), (5.1.9), and (5.1.10) we see that: 


$$
(-1)^{r} \sum_{n=1}^{\infty} Y_{n}^{(\alpha)}(P) \Delta^{(\alpha+1)}\left\{\frac{P_{n}(\cos h)}{[n(n+1)]^{r}}\right\}=\sum_{k=0}^{r} a_{k}(1-\cos h)^{k}+L^{r}(\cos h)
$$

where

$$
\begin{aligned}
& a_{0}=(-1)^{r} \sum_{n=1}^{\infty} Y_{n}^{(\alpha)}(P) \Delta^{(\alpha+1)}\left\{\frac{1}{[n(n+1)]^{r}}\right\}, \\
& a_{k}=(-1)^{r} \sum_{n=1}^{\infty} Y_{n}^{(\alpha)}(P) \Delta^{(\alpha+1)}\left\{\frac{\Delta_{0}\left(\Delta_{0}+1 \cdot 2\right) \cdots\left[\Delta_{0}+(k-1) k\right] P_{n}}{[n(n+1)]^{r}}\right\}, \\
& k=1, \cdots, r .
\end{aligned}
$$

But from [5, p. 128, Theorem 1] we know that if (i) $\sum b_{n}$ is summable or bounded $(C-k), k$ an integer, (ii) $F_{n} \rightarrow 0$, and (iii) $\sum(n+1)^{k}\left|\Delta^{(k+1)} F_{n}\right|<\infty$, then $\sum b_{n} F_{n}$ is summable $(C-k)$ to $\sum B_{n}^{(k)} \Delta^{(k+1)} F_{n}$ the last series being absolutely convergent. It is to be noted that the theorem is valid if (ii) is replaced by (ii') $F_{n}=K+o(1)$, where $K$ is a positive constant. With $F_{n}$ $\equiv 1 /[n(n+1)]^{r}$ for $k=0, F_{n} \equiv \Delta_{0}\left(\Delta_{0}+1 \cdot 2\right) \cdots\left[\Delta_{0}+(k-1) k\right] P_{n} /[n(n+1)]^{r}$ for $k=1, \cdots, r$, and $b_{n} \equiv Y_{n}(P)$ it is obvious that the hypotheses (i), (ii'), and (iii) of the above theorem are satisfied. Consequently we have:

$$
\begin{aligned}
& a_{0}=\text { the }(C-\alpha) \text { sum of the series }(-1)^{r} \sum_{n=1}^{\infty} Y_{n}(P) \cdot \frac{1}{[n(n+1)]^{r}}, \\
& a_{k}=\text { the }(C-\alpha) \text { sum of the series } \\
&(-1)^{r} \sum_{n=1}^{\infty} Y_{n}(P) \frac{\Delta_{0}\left(\Delta_{0}+1 \cdot 2\right) \cdots\left[\Delta_{0}+(k-1) k\right] P_{n}}{[n(n+1)]^{r}}, k=1, \cdots, r .
\end{aligned}
$$

and the proof of the lemma is complete.

5.2. Lemma 2. Let $R_{n}^{r}(\cos h)$ be defined by:

$$
\begin{aligned}
R_{n}^{r}(\cos h) \equiv & P_{n}(\cos h) \\
& -\left\{1+\Delta_{0} P_{n} \frac{(1-\cos h)}{2}+\frac{\Delta_{0}\left(\Delta_{0}+1 \cdot 2\right) P_{n}}{(2 !)^{2}} \frac{(1-\cos h)^{2}}{2^{2}}+\cdots\right. \\
& \left.+\frac{\Delta_{0}\left(\Delta_{0}+1 \cdot 2\right) \cdots\left[\Delta_{0}+(r-1) r\right] P_{n}}{(r !)^{2}} \frac{(1-\cos h)^{r}}{2^{r}}\right\}
\end{aligned}
$$

where $0<h<\pi$ and $r$ is a positive integer. If $j$ is an integer such that $(j+1) / 2<r$ and $n \leqq\left[(1 /(1-\cos h))^{1 / 2}\right]$, then there exists a positive constant $K$ (independent of $r, n$, and $h$ ) such that:

$$
\left|\Delta^{(j)} \frac{R_{n}^{r}(\cos h)}{(1-\cos h)^{r}}\right|<K n^{2(r+1)-j(1-\cos h) .}
$$


Proof. We may assume that $n \geqq r+1$ for if $n \leqq r, \Delta_{0}\left(\Delta_{0}+1 \cdot 2\right) \cdots$ $\left[\Delta_{0}+(k-1) k\right] P_{n}=0$ for $n \leqq k \leqq r$. Thus $R_{n}^{r}(\cos h)=0$ and $\Delta^{(j)}\left\{R_{n}^{r}(\cos h) /(1-\cos h)^{r}\right\}=0$. Employing the expansion for $P_{n}(\cos h)$ we see that:

$$
\begin{aligned}
& \frac{R_{n}^{r}(\cos h)}{(1-\cos h)^{r+1}}=(-1)^{r+1} \frac{(n-r) \cdots n(n+1) \cdots(n+r+1)}{[(r+1) !]^{2}} \cdot \frac{1}{2^{r+1}}+\cdots \\
& +(-1)^{k+r+1} \frac{[n-(k+r)] \cdots n(n+1) \cdots[n+(k+r)+1]}{[(k+r+1) !]^{2}} \\
& .2 .2) \\
& +(-1)^{n} \frac{(1-\cos h)^{k}}{2^{k+(r+1)}}+\cdots \\
& (n !)^{2}
\end{aligned}
$$

But since (see $[1$, p. $6,(3)]) \Delta^{(j)} x^{(m)}=m(m-1) \cdots(m-j+1) x^{(m-j)}$ where $x^{(m)} \equiv x(x-1) \cdots(x-m+1)$ we see, letting $x=n+k+r+1$ and $m=2(k+r+1)$, that:

$$
\begin{aligned}
& \Delta^{(j)}\left\{\frac{[n-(k+r)] \cdots n(n+1) \cdots[n+(k+r)+1]}{[(k+r+1) !]^{2}} \frac{(1-\cos h)^{k}}{2^{k+r+1}}\right\} \\
& =\frac{2^{j}[k+r+1]\left[k+r+\frac{1}{2}\right] \cdots\left[k+r+1-\left(\frac{j-1}{2}\right)\right]}{[(k+r+1) !]^{2}} \\
& \cdot(n+k+r+1) \cdots(n+1) n \cdots[n-(k+r)+j] \frac{(1-\cos h)^{k}}{2^{k+r+1}} \\
& \text { for } k=0,1, \cdots, n-(r+1) .
\end{aligned}
$$

Since $(n+k+r+1) \cdots(n+1)$ is, for $k=0,1, \cdots, n-(r+1)$, clearly majorized by $(2 n)^{k+r+1}$ and $n \cdots[n-(k+r)+j]$ by $n^{k+r+1-i}$, for $n^{2}(1-\cos h) \leqq 1$ we have the expression on the right of (5.2.3) majorized by $\left\{2^{i} /[1 \cdot 2 \cdot \cdots \cdot((k+r)-(j+1) / 2)]^{2}\right\} n^{2(r+1)-j}$. But for $r>(j+1) / 2$, this expression is in turn majorized by $\left[2^{j} /(k !)^{2}\right] n^{2(r+1)-j}$. Hence from (5.2.2) and (5.2.3) there exists a positive constant $K$ such that:

$$
\begin{aligned}
& \left|\Delta^{(j)}\left\{\frac{R_{n}^{r}(\cos h)}{(1-\cos h)^{r+1}}\right\}\right| \\
& \equiv \mid \sum_{k=0}^{n-(r+1)} \Delta^{(j)}\left\{(-1)^{k} \frac{[n-(k+r)] \cdots n(n+1) \cdots[n+(k+r)+1]}{\left[\left(k+r_{-}+1\right) !\right]^{2}}\right. \\
& \left.\cdot \frac{(1-\cdots \cos h)^{k}}{2^{k+r+1}}\right\} \mid<K n^{2(r+1)-j}
\end{aligned}
$$


The lemma then follows immediately.

5.3. Lemma 3. Let $P_{n}(\cos h)$ be the Legendre polynomials of order $n, n$ $=1,2, \cdots$. If $n>[1 /(1-\cos h)]^{1 / 2}$ where $0<h<\pi$ then for any non-negative integer $r$ :

$$
\Delta^{(r)} P_{n}(\cos h)=O\left[n(1-\cos h)^{(r+1) / 2}\right] .
$$

Proof. The proof is by induction. The case $r=0$ is trivial since $\left|P_{n}(\cos h)\right|$ $\leqq 1$ and $n(1-\cos h)^{1 / 2}>1$. Assuming that

$$
\Delta^{(m)} P_{n}(\cos h)=O\left[n(1-\cos h)^{(m+1) / 2}\right] \quad \text { for } m=0,1, \cdots, k,
$$

it will be shown that $\Delta^{(k+1)} P_{n}(\cos h)=O\left[n(1-\cos h)^{(k+2) / 2}\right]$. From the Christoffel-Darboux formula [2, p. 159] we know that:

$$
\frac{\Delta P_{n}(\cos h)}{(1-\cos h)}=\frac{K}{n+1} \sum_{j=0}^{n}(j+1 / 2) P_{j}(\cos h)
$$

where $K$ is a positive constant. Thus it is easily seen from (5.3.2) that:

$$
\begin{aligned}
\Delta^{(m-1)}\left\{\frac{1}{n+1} \sum_{j=0}^{n}(j+1 / 2) P_{j}(\cos h)\right\}=O\left[n(1-\cos h)^{(m-1) / 2}\right] & \\
& \text { for } m=1, \cdots, k .
\end{aligned}
$$

Also, utilizing (5.3.3),

$$
\begin{aligned}
\Delta^{(k+1)}\left\{\frac{P_{n}(\cos h)}{(1-\cos h)}\right\}= & K \Delta^{(k-1)}\left\{\Delta\left[\frac{1}{(n+1)} \sum_{j=0}^{n}(j+1 / 2) P_{j}(\cos h)\right]\right\} \\
= & K\left[\Delta^{(k-1)}\left\{-\frac{n+3 / 2}{n+1} P_{n+1}(\cos h)\right\}\right. \\
& \left.+\Delta^{(k-1)}\left\{\frac{1}{n+2} \cdot \frac{1}{n+1} \sum_{j=0}^{n+1}(j+1 / 2) P_{j}(\cos h)\right\}\right] .
\end{aligned}
$$

Consequently, from (5.1.3), the induction hypothesis (5.3.2), and (5.3.4), we have:

$$
\begin{aligned}
\Delta^{(k+1)}\left\{\frac{P_{n}(\cos h)}{(1-\cos h)}\right\} & \\
= & O\left[n\left\{\sum_{j=0}^{k-1}\left(\begin{array}{c}
k-1 \\
j
\end{array}\right) \frac{(1-\cos h)^{(j+1) / 2}}{n^{k-1-j}}+\frac{(1-\cos h)^{j / 2}}{n^{k-j}}\right\}\right] .
\end{aligned}
$$

Therefore, since $1 / n<(1-\cos h)^{1 / 2}$,

$$
\Delta^{(k+1)}\left\{\frac{P_{n}(\cos h)}{(1-\cos h)}\right\}=O\left[n(1-\cos h)^{k / 2}\right],
$$

and the proof is complete. 
We now proceed to state and prove the necessary conditions for $C$ summability.

5.4. Theorem. Let $\sum_{n=0}^{\infty} Y_{n}(Q)$ be a series of surface spherical harmonics on $\Omega$. Let $r$ be a non-negative integer great enough so that $\Delta^{(-r)}\left\{\sum_{n=0}^{\infty} Y_{n}(Q)\right\}$ converges uniformly to $F_{r}(Q)$ on $\Omega$. If $\sum_{n=0}^{\infty} Y_{n}(Q)$ is $(C-\alpha)$ summable, $\alpha$ a non-negative integer, to $s$ at the point $P$ on $\Omega$ then for $r$ an integer greater than $(\alpha+2) / 2, \triangle_{r} F_{r}(P)$ exists and equals $s$.

Proof. Without loss of generality we may assume that $Y_{0}=0$ and $\sum_{n=1}^{\infty} Y_{n}(Q)$ is $(C-\alpha)$ summable to 0 at $P$. Since $\Delta^{(-r)}\left\{\sum_{n=1}^{\infty} Y_{n}(Q)\right\}$ converges uniformly to $F_{r}(Q)$, by $[7$, p. 298] it is to be seen that:

$$
\frac{1}{2 \pi \sin h} \int_{C(P, h)} F_{r}(Q) d s_{Q}=(-1)^{r} \sum_{n=1}^{\infty} \frac{Y_{n}(P) P_{n}(\cos h)}{[n(n+1)]^{r}} .
$$

Applying Abel's partial summation formula $(\alpha+1)$ times to the right side of this equation we obtain:

$$
\frac{1}{2 \pi \sin h} \int_{C(P, h)} F_{r}(Q) d s_{Q}=(-1)^{r} \sum_{n=1}^{\infty} Y_{n}^{(\alpha)}(P) \Delta^{(\alpha+1)}\left\{\frac{P_{n}(\cos h)}{[n(n+1)]^{r}}\right\} .
$$

Note that the hypothesis for the application of this formula is satisfied since $Y_{n}^{(k)}(P)=o\left(n^{\alpha}\right)$ for $k=0, \cdots, \alpha$, and as shown in $\$(5.1 .4)$, for $r>(\alpha+1) / 2$, $\Delta^{(k+1)}\left\{P_{n}(\cos h) /[n(n+1)]^{r}\right\}=O\left(1 / n^{2 r}\right) \quad$ for $\quad k=0, \cdots, \alpha$. Thus $\sum_{n=1}^{\infty} Y_{n}^{(\mathbf{k})}(P) \Delta^{(k+1)}\left\{P_{n}(\cos h) /[n(n+1)]^{r}\right\} \quad$ converges absolutely and $Y_{n}^{(k)}(P) \Delta^{(k+1)}\left\{P_{n}(\cos h) /[n(n+1)]^{r}\right\}=o(1)$ for $k=0, \cdots, \alpha$. Applying (5.1.1) to the right member of (5.4.1) and comparing the result with (2.12) and the definition (2.1.1), it is seen that if $L_{r}(\cos h)=o(1-\cos h)^{r}$ then:

$$
\begin{aligned}
& \frac{1}{2 \pi \sin h} \int_{C(P, h)} F_{r}(Q) d s_{Q}=\Delta_{0} F_{r}(P)+\triangle_{1} F_{r}(P) \frac{(1-\cos h)}{2} \\
& +\frac{\Delta(\Delta+1 \cdot 2) F_{r}(P)}{(2 !)^{2}} \frac{(1-\cos h)^{2}}{2^{2}}+\cdots \\
& +\frac{\Delta(\Delta+1 \cdot 2) \cdots[\Delta+(r-1) r] F_{r}(P)}{(r !)^{2}} \frac{(1-\cos h)^{r}}{2^{r}}+o(1-\cos h)^{r}
\end{aligned}
$$

where $\Delta_{k} F_{r}(P), k=0, \cdots, r$ are defined uniquely by the following set of $(r+1)$ equations:

$$
\triangle_{0} F_{r}(P)=\text { The }(C-\alpha) \text { sum of the series }(-1)^{r} \sum_{n=1}^{\infty} Y_{n}(P) \frac{1}{[n(n+1)]^{r}},
$$

(5.4.3) $\Delta(\Delta+1 \cdot 2) \cdots[\Delta+(k-1) k] F_{r}(P)=$ The $(C-\alpha)$ sum of the series

$$
(-1)^{r} \sum_{n=1}^{\infty} Y_{n}(P) \frac{\Delta_{0}\left(\Delta_{0}+1 \cdot 2\right) \cdots\left[\Delta_{0}+(k-1) k\right] P_{n}}{[n(n+1)]^{r}}, \quad k=1, \cdots, r \text {. }
$$


Thus $\Delta_{r} F_{r}(P)$ will be equal to the $(C-\alpha)$ sum of

$$
(-1)^{r} \sum_{n=1}^{\infty} Y_{n}(P) \Delta_{0}^{(r)} P_{n}(\cos h) /[n(n+1)]^{r}=\sum_{n=1}^{\infty} Y_{n}(P) .
$$

We therefore need only show that

$$
\begin{array}{rlrl}
L^{r}(\cos h) & \equiv[1 / 2 \pi \sin h] \int_{C(P, h)} F_{r}(Q) d s_{Q}-\left\{\Delta_{0} F_{r}(P)+\sum_{k=0}^{r}[\Delta(\Delta+1 \cdot 2) \cdots\right. \\
& =o(1-\cos h)^{r} & \left.\left.\cdot[\Delta+(k-1) k] F_{r}(P) /(k !)^{2}\right](1-\cos h)^{k} / 2^{k}\right\}
\end{array}
$$

where $\Delta_{k} F_{r}(P), k=0, \cdots, r$, are given by (5.4.3). From (5.4.1), (5.1.14), and (5.1.15) we have:

$L^{r}(\cos h)$

$$
=(-1)^{r}(1-\cos h)^{r} \sum_{n=1}^{\infty} Y_{n}^{(\alpha)}(P) \Delta^{(\alpha+1)}\left\{\frac{R_{n}^{r}(\cos h)}{[n(n+1)]^{r}(1-\cos h)^{r}}\right\}
$$

where

$$
\begin{gathered}
R_{n}^{r}(\cos h) \equiv P_{n}(\cos h) \\
(5.4 .5)-\left[1+\Delta_{0}^{(1)} P_{n} \frac{(1-\cos h)}{2}+\frac{\Delta_{0}\left(\Delta_{0}+1 \cdot 2\right) P_{n}}{(2 !)^{2}} \frac{(1-\cos h)^{2}}{2^{2}}+\cdots\right. \\
\left.\quad+\frac{\Delta_{0}\left(\Delta_{0}+1 \cdot 2\right) \cdots\left[\Delta_{0}+(r-1) r\right] P_{n}}{(r !)^{2}} \frac{(1-\cos h)^{r}}{2^{r}}\right] .
\end{gathered}
$$

It thus only remains to show that

$$
\sum_{n=1}^{\infty} Y_{n}^{(\alpha)}(P) \Delta^{(\alpha+1)}\left\{R_{n}^{r}(\cos h) /[n(n+1)]^{r}(1-\cos h)^{r}\right\}
$$

is $o(1)$. Let

$$
\begin{aligned}
\sum_{n=1}^{\infty} Y_{n}^{(\alpha)}(P) \Delta^{(\alpha+1)} & \left\{\frac{R_{n}^{r}(\cos h)}{[n(n+1)]^{r}(1-\cos h)^{r}}\right\} \\
= & \sum_{n=1}^{N} Y_{n}^{(\alpha)}(P) \Delta^{(\alpha+1)}\left\{\frac{R_{n}(\cos h)}{[n(n+1)]^{r}(1-\cos h)^{r}}\right\} \\
& +\sum_{n=N+1}^{\infty} Y_{n}^{(\alpha)}(P) \Delta^{(\alpha+1)}\left\{\frac{R_{n}^{r}(\cos h)}{[n(n+1)]^{r}(1-\cos h)^{r}}\right\} \text { where } \\
N= & {\left[\left(\frac{1}{1-\cos h}\right)^{1 / 2}\right] . }
\end{aligned}
$$


Applying (5.1.3) to $R_{n}^{r}(\cos h) /[n(n+1)]^{r}(1-\cos h)^{r}$ with

$$
f_{n} \equiv R_{n}^{r}(\cos h) /(1-\cos h)^{r} \quad \text { and } g_{n} \equiv 1 /[n(n+1)]^{r}
$$

we see from (5.2.1) Lemma 2 that for $n \leqq N$ there exists a $K>0$ such that $\left|\Delta^{(j)}\left\{R_{n}^{r}(\cos h) /[n(n+1)]^{r}(1-\cos h)^{r}\right\}\right|<K n^{(1-\alpha)}(1-\cos h)$. Consequently,

$$
\sum_{n=1}^{N} Y_{n}^{(\alpha)}(P) \Delta^{(\alpha+1)}\left\{\frac{R_{n}^{r}(\cos h)}{[n(n+1)]^{r}(1-\cos h)^{r}}\right\}
$$

$$
=o\left[N^{2}(1-\cos h)\right]=o(1) .
$$

Again applying (5.1.3) to $P_{n}(\cos h) /[n(n+1)]^{r}(1-\cos h)^{r} \quad$ with $f_{n} \equiv P_{n}(\cos h) /(1-\cos h)^{r}$ and $g_{n} \equiv 1 /[n(n+1)]^{r}$ we see from (5.3.1) Lemma 3 that for $n>N$,

$$
\begin{aligned}
\Delta^{(\alpha+1)}\left\{P_{n}(\cos h) /[n(n+1)]^{r}(1\right. & \left.-\cos h)^{r}\right\} \\
& =O\left[1 / n^{(2 r-1)}(1-\cos h)^{r-((\alpha+2) / 2)}\right] .
\end{aligned}
$$

Consequently,

$$
\begin{aligned}
& \sum_{n=N+1}^{\infty} Y_{n}^{(\alpha)}(P) \Delta^{(\alpha+1)}\left\{\frac{P_{n}(\cos h)}{[n(n+1)]^{r}}\right\} \\
& \quad=o\left[\frac{1}{N^{2}(1-\cos h)^{r((\alpha+2) / 2)}}\right]=o(1) \quad \text { for } r>(\alpha+2) / 2 .
\end{aligned}
$$

Now for $k=1, \cdots, r$,

$$
\begin{aligned}
\Delta^{(\alpha+1)}\left\{\frac{\frac{\Delta_{0}\left(\Delta_{0}+1 \cdot 2\right) \cdots\left[\Delta_{0}+(k-1) k\right] P_{n}}{(k !)^{2}} \frac{(1-\cos h)^{k}}{2^{k}}}{[n(n+1)]^{r}(1-\cos h)^{r}}\right\} \\
=O\left[\frac{1}{n^{2(r-k)+\alpha+1}(1-\cos h)^{r-k}}\right]
\end{aligned}
$$

since for $j=1, \cdots, k ; k=1, \cdots, r$, and $j \neq r$,

$$
\Delta^{(\alpha+1)}\left\{\frac{\frac{\Delta_{0}^{(j)} P_{n}}{(k !)^{2}} \frac{(1-\cos h)^{k}}{2^{k}}}{[n(n+1)]^{r}(1-\cos h)^{r}}\right\}=O\left[\frac{1}{n^{2(r-k)+\alpha+1}(1-\cos h)^{r-k}}\right]
$$

and for $j=k=r$, 
Thus,

$$
\Delta^{(\alpha+1)}\left\{\frac{\frac{\Delta_{0}^{r} P_{n}}{(r !)^{2}} \frac{(1-\cos h)^{r}}{2^{r}}}{[n(n+1)]^{r}(1-\cos h)^{r}}\right\}=\Delta^{(\alpha+1)}\left\{\frac{(-1)^{r}}{2^{r}(r !)^{2}}\right\}=0 .
$$

$$
\begin{aligned}
& \sum_{n=1} Y_{n}^{(\alpha)}(P) \Delta^{(\alpha+1)} \\
& \left\{\begin{array}{c}
1+\Delta_{0}^{(1)} P_{n} \frac{(1-\cos h)}{2}+\frac{\Delta_{0}\left(\Delta_{0}+1 \cdot 2\right) P_{n}}{(2 !)^{2}} \frac{(1-\cos h)^{2}}{2^{2}}+\cdots \\
+\frac{\Delta_{0}\left(\Delta_{0}+1 \cdot 2\right) \cdots\left[\Delta_{0}+(r-1) r\right] P_{n}}{(r !)^{2}} \frac{(1-\cos h)^{r}}{2^{r}}
\end{array}\right\} \\
& =o\left[\frac{1}{\left.\left.(1-\cos h)^{r-k}(N+1)^{2(r-k)}\right]\right]^{r}(1-\cos h)^{r}}\right]=o(1),
\end{aligned}
$$

and from (5.4.6), (5.4.7), (5.4.8), and (5.4.9) it follows that for $r>(\alpha+2) / 2$, $\sum_{n=1}^{\infty} Y_{n}^{(\alpha)}(P) \Delta^{(\alpha+1)}\left\{R_{n}^{r}(\cos h) /[n(n+1)]^{r}(1-\cos h)^{r}\right\}=o(1)$.

As a consequence of this theorem we can prove the following result:

5.5. Theorem. If $f(Q) \in C^{2 r}, r=1,2, \cdots$, in a neighborhood of any point $P$ on $\Omega$ then $\Delta_{r} f(P)$ exists and $\Delta_{k} f(P)=\Delta^{(k)} f(P), k=1, \cdots, r$.

Proof. By hypothesis there exists a spherical cap $I(P, h) \equiv\{Q \mid[Q, P]$ $>\cos h, 0<h<\pi\}$ on which $f(Q) \in C^{2 r}$. Let $\psi$ be a localizing function of class $C^{\infty}$ on $\Omega$ for the spherical caps $I\left(P, h_{1}\right), I\left(P, h_{2}\right)$ where $0<h_{1}<h<h_{2}$. Then $\psi f(Q) \in C^{2 r}$ on $\Omega$. Let $S[\psi f(Q)]$ be the Laplace series of $\psi f(Q)$ where without loss of generality we may assume that the constant term is zero. $S\left[\Delta^{(k)} \psi f(Q)\right]$ $=\Delta^{(k)} S[\psi f(Q)], k=1, \cdots, r$, for by $\left[8\right.$, p. 213] if $F_{1}(Q)$ and $F_{2}(Q) \in C^{2}$ on $\Omega$ then Green's second identity holds, i.e.,

$$
\iint_{\Omega} F_{1}(Q) \Delta F_{2}(Q) d \Omega_{Q}=\iint_{\Omega} \Delta F_{1}(Q) F_{2}(Q) d \Omega_{Q}
$$

Thus since $\psi f(Q)$ and $P_{n}([M, Q]) \in C^{2 r}$ on $\Omega$, this identity may be applied $r$ times to $\iint_{\Omega} \psi f(M) P_{n}([M, Q]) d \Omega_{M}, n=1,2, \cdots$, to yield the above result. Since $\Delta^{(k)} \psi f(Q)$ is continuous on $\Omega, S\left[\Delta^{(k)} \psi f(Q)\right] \equiv \Delta^{(k)} S[\psi f(Q)]$ is $(C-1)$ summable to $\Delta^{(k)} \psi f(Q)$ on $\Omega$ (see [4]). Each term of $S\left[\Delta^{(k)} \psi f(Q)\right]$ is $o(n)$ by the $(C-1)$ summability. Thus applying the theorem of the preceding section to $\Delta^{(k)} S[\psi f(Q)]$ for $k=2, \cdots, r ; r \geqq 2$, we form $\Delta^{(-k)}\left\{\Delta^{(k)} S[\psi f(Q)]\right\}$ $\equiv S[\psi f(Q)]$ and conclude that $\Delta_{k} S[\psi f(Q)]$ exists and equals $\Delta^{(k)} \psi f(Q)$. Since $\psi f(Q) \in C^{2 r}, S[\psi f(Q)]$ converges to $\psi f(Q)$. Thus $\Delta_{k} \psi f(Q)=\Delta^{(k)} \psi f(Q)$ and since $\psi=1$ in $I\left(P, h_{1}\right), \Delta_{k} f(P)=\Delta^{(k)} f(P)$ for $k=2, \cdots, r ; r \geqq 2$. If $\Delta_{1} f(P)$ exists then by the theorem of $\$ 4.11, \triangle^{(1)} S[\psi f(Q)]$ is $(C-\alpha)$ summable at $P$, $\alpha>3$, to $\Delta_{1} f(P)$. Thus $\Delta_{1} f(P) \Delta^{(1)} f(P)$. If $r \geqq 2$, the existence of $\Delta_{1} f(P)$ 
follows immediately from that of $\Delta_{k} f(P)$ for $k=2, \cdots, r$. If $r=1$, that $f \in C^{2}$ in $I(P, h)$ implies the existence of $\triangle_{1} f(P)$ is shown as follows: By definition, $f(Q) \in C^{2}$ in $I(P, h) \Leftrightarrow g(x, y) \in C^{2}$ on $\left\{(x, y) \mid x^{2}+y^{2}<\sin ^{2} h\right\}$ where $g(x, y) \equiv f\left(x, y,\left(1-x^{2}-y^{2}\right)^{1 / 2}\right)$ and $f(x, y, z) \equiv f(Q)$ for all points $Q$ in $I(P, h)$. Then by Taylor's Theorem, for $0<t<h,(1 / 2 \pi \sin t) \int_{C(P, t)} f(Q) d s_{Q}$ $=(1 / 2 \pi) \int_{0}^{2 \pi} g(\sin t \cos \theta, \sin t \sin \theta) d \theta=g(0,0)+(1 / 4)\left[g_{x x}(0,0)+g_{y y}(0,0)\right] \sin ^{2} t$ $+o\left(\sin ^{2} t\right)$. Thus:

$$
\lim _{t \rightarrow 0} \frac{(1 / 2 \pi \sin t) \int_{C(P, t)} f(Q) d s_{Q}-f(P)}{(1-\cos t)}=(1 / 2)\left[g_{x x}(0,0)+g_{y y}(0,0)\right]
$$

and consequently $\Delta_{1} f(P)$ exists and in fact equals $\left[g_{x x}(0,0)+g_{y y}(0,0)\right]$.

VI. The necessary and sufficient conditions for $C$ summability.

THEOREM. Let $\sum_{n=0}^{\infty} Y_{n}(Q)$ be a series of surface spherical harmonics with $Y_{n}(Q)=O\left(n^{k}\right)$ uniformly on $\Omega$ for some $k . A$ necessary and sufficient condition that $\sum_{n=0}^{\infty} Y_{n}(Q)$ be summable $C$ to $s$ at an arbitrary point $P$ on $\Omega$ is that there exist a non-negative integer $r>(k+1) / 2$ such that $\triangle_{r} F_{r}(P)$ exists and equals $s$ where $F_{r}(Q) \equiv \Delta^{(-r)}\left\{\sum_{n=0}^{\infty} Y_{n}(Q)\right\}$.

Proof. The sufficiency follows immediately from the theorem of $\$ 4.12$ with the order of summability $\alpha>2 r+1$. Choosing $r$ an integer greater than $\max \{(k+1) / 2,([\alpha]+3) / 2\}$ where $\alpha$ is the order (not necessarily integral) of summability of $\sum_{n=0}^{\infty} Y_{n}(Q)$ at $P$, the necessity follows immediately from the theorem of $\$ 5.4$.

\section{BIBLIOGRAPHY}

1. G. Boole, Calculus of finite differences, Chelsea, New York, 1860.

2. A. Erdélyi, W. Magnus, F. Oberhettinger, and F. G. Tricomi, Higher transcendental functions, Vol. 2, McGraw-Hill, New York, 1953.

3. L. Fejer, Über die Summabilitat der Laplaceschen Reihe durch arithmetische Mittel, Math. Z. 24(1926), 267-284.

4. T. H. Gronwall, Über die Laplaceshe Reihe, Math. Ann. 74 (1913), 213-270.

5. G. H. Hardy, Divergent series, Clarendon Press, Oxford, 1949.

6. E. W. Hobson, The theory of spherical and ellipsoidal harmonics, Cambridge Univ. Press, London, 1931. 303.

7. W. Rudin, Unigueness theory for Laplace series, Trans. Amer. Math. Soc. 68 (1950), 287-

8. V. L. Shapiro, Localization on spheres, Trans. Amer. Math. Soc. 86 (1957), 212-219.

9. - The symmetric derivative on the $(k-1)$ dimensional hypersphere, Trans. Amer. Math. Soc. 81 (1956), 514-524.

10. W. J. Sternberg and T. L. Smith, The theory of the potential and spherical harmonics, Univ. of Toronto Press, Toronto, 1946.

11. A. Zygmund, Trigonometrical series, Warsaw, 1935.

College of South Jersey, Rutgers University, Camden, New Jersey

Drexel Institute of TeChNology, Puiladelphia, Pennsylvania 